\title{
AN EMPIRICAL STUDY WITH A LOW-COST STRATEGY FOR IMPROVING THE ENERGY DISAGgREGATION VIA QUESTIONNAIRE SURVEY
}

\author{
Chun-peng Chang, Wen-Jen Ho, Yung-chieh Hung, \\ Kuei-Chun Chiang and Bill Zhao \\ Institute for Information Industry, Taipei, Taiwan
}

\begin{abstract}
Based on neural network and machine learning, we apply the energy disaggregation for both classification (prediction on usage time) and estimation (prediction on usage amount) on 150 AMI (Advanced Metering Infrastructure) smart meters and a small amount of HEMS (Home Energy Management System) smart plugs in a community in New Taipei City, Taiwan. The aim of this paper is to clarify how we lower the cost, obtain the model of appliance usage from only a small portion of households, improve it with simple questionnaire, and generalize it for prediction on collective households. Our investigation demonstrates the benefits and various possibilities for power suppliers and the government, and won the Elite Award in the Presidential Hackathon 2020, Taiwan.
\end{abstract}

\section{KEYWORDS}

Energy Disaggregation, Non-intrusive Load Monitoring, Deep Learning, Autoencoder

\section{INTRODUCTION}

The big data of electricity sales services will be used to provide users with more various valueadded applications and power suppliers with business opportunities. Energy disaggregation, or so called NILM (Non-intrusive Load Monitoring), is a particular study field in the electricity industry, and has huge potential to benefit targets mentioned above. It was developed by George W. Hart [1] in the 80s, to infer the individual states of the appliances from the aggregated meter measuring the voltage and the current from outside the houses. This is exactly the literal meaning of "Non-intrusive" in NILM. Nowadays, it is not only a theoretical study, but also a practical strategy going to start in many countries.

A recent research work of Kelly and Knottenbelt [2] have demonstrated the possibility of utilizing deep learning, which leads successful progress in many fields, such as image recognition, into the region of NILM. Hereafter, many researches in energy disaggregation [3] was developed quickly. These researches, however, are not suitable for numerous households outside the laboratory due to both the price and the privacy. Expensive meters with high sampling rate are needed for every appliance inside the house, and hence not applicable for a generalization to the whole city or the whole country. On the other hand, AMI, the cheap smart meters outside the house with low-sampling rate of $1 \mathrm{mHz}$ (sampling period of $15 \mathrm{~min}$ ) [4][5], are quite suitable. And more and more countries regard AMI as fundamental infrastructure. Our 
study is based on Ming-Hsuan-Huang-Cheng, a real community in New Taipei City, Taiwan. This community is of 150 households, in which all are with AMI outside their houses. Moreover, within this community we have collected 20 volunteer households and deployed smart plugs of HEMS inside each of their house, for up to five appliances (air conditioner, refrigerator, washing machine, bottle warmer, and television) and the total power. We have collected the HEMS data of these volunteers for 1 year so far. This study focuses on the period of June 2020, for both AMI and HEMS data, and make classification and estimation on the 150 AMI households with their AMI meters only.

For the reference, we provided a visual example of AMI and HEMS data, as figure 1 and 2.

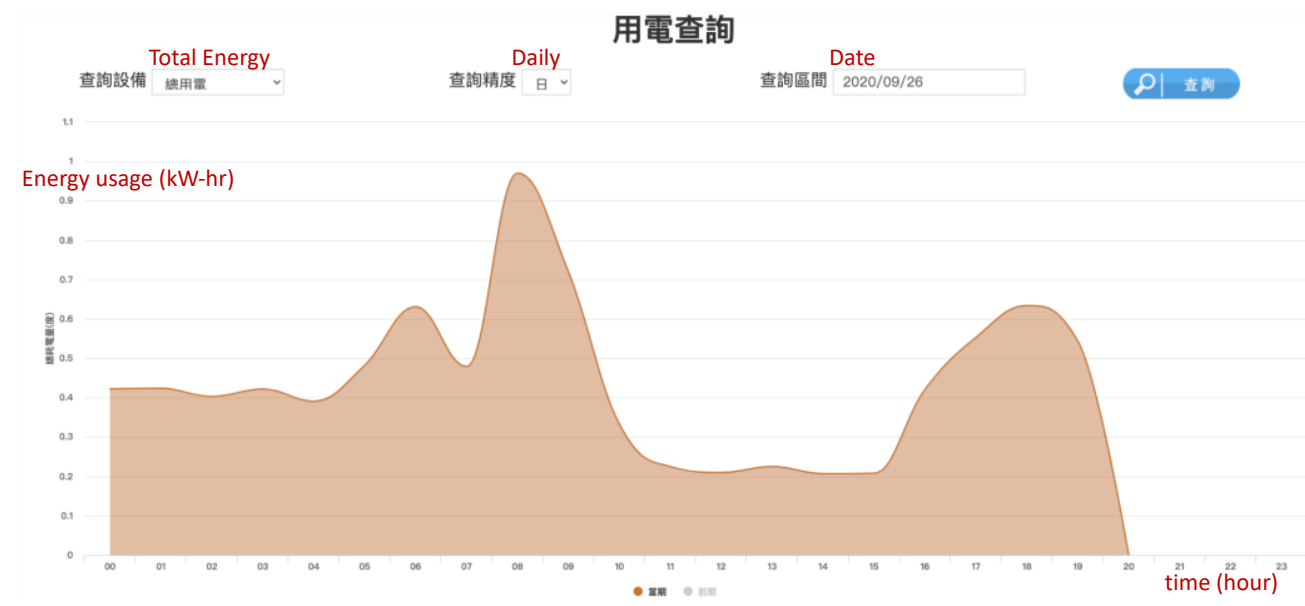

Figure 1: A visual example of total energy consumption in AMI data.

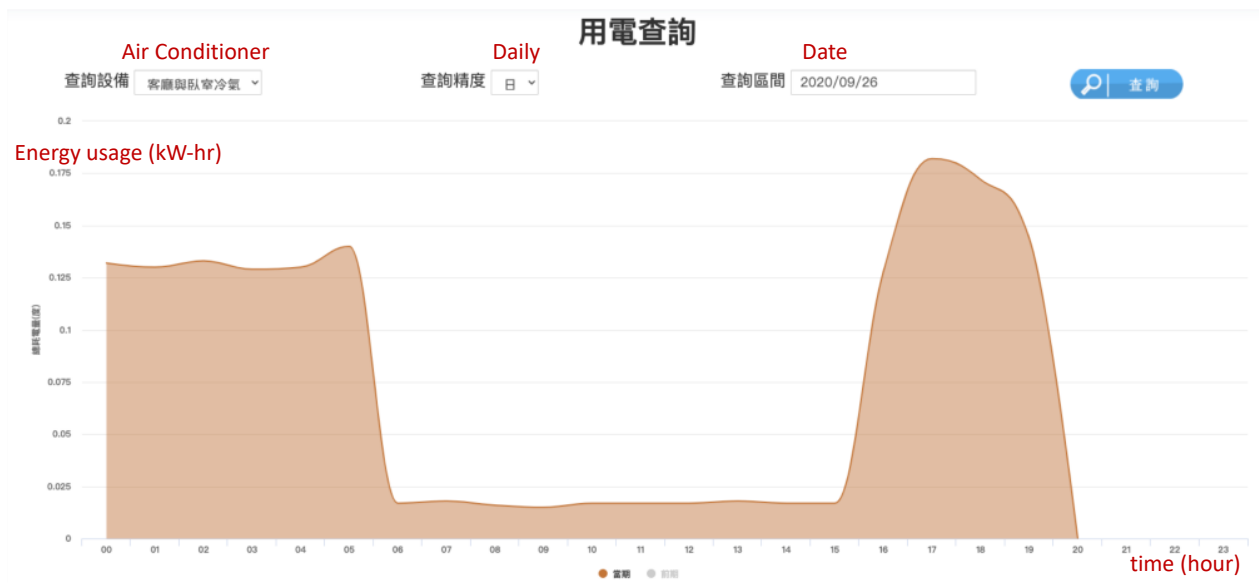

Figure 2: A visual example of energy consumption of the air conditioner in HEMS data.

\section{METHOD}

This study is composed of 2 parts: NILM estimation and classification.

\subsection{NILM estimation}

The first part, estimation, is to estimate the portion of energy consumed owing to each appliance. The estimation strategy is inspired by our previous research "An Analysis of Semi-Supervised 
Learning Approaches in Low-Rate Energy Disaggregation" [5] and imitate a semi-supervised learning framework similar to it. As Figure 3, we perform sparse auto-encoder for the feature extraction on the daily time series of total power of both AMI and HEMS, and cluster these features with K-means clustering so that each HEMS feature is correspond to some ones of AMI nearby in the feature space. Through clustering we may naturally assume that the usage behaviors in the same cluster are similar, so we assign appliance consumption of HEMS as labels to the total power data of AMI in the same cluster, which lack these labels originally. This process is the unsupervised learning stage to obtain the feature extraction models. Hereafter, the sample range for subsequent supervised training is enlarged from 20 HEMS households to 150 AMI households. The main weakness of supervised learning on few samples is overcome.
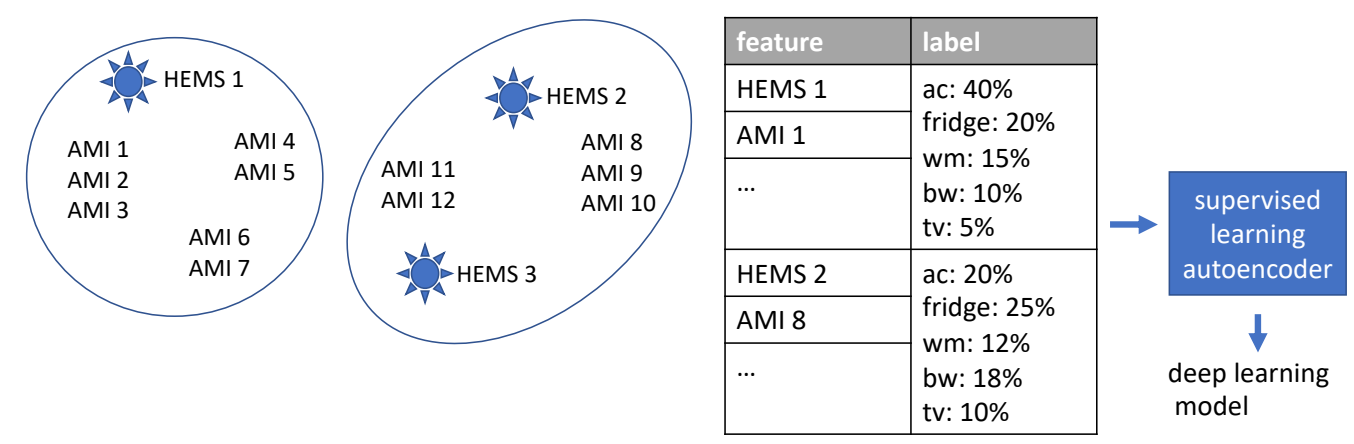

Figure 3: training process for the estimation model. By clustering features in the feature space, we assume features in the same cluster should have similar appliance consumption.

\subsection{NILM classification}

On the other hand, the second part of this study, classification, is to determine whether each appliance is turned on in a period of time. We divide a day into 3 sections: morning 7:00 12:00, afternoon 12:00 22:00, and night 22:00 7:00 as the classification labels. This partition is designed to match the behavior of common households. We build a multi-label binary classifier to classify the daily usage data. For refrigerators, which are supposed be always turned on, we focus on the period when they are heavily used.

\section{EXPERIMENTS}

We divide 20 HEMS volunteer households for 5-fold cross validation. In each turn 16 households are for the training data and 4 ones are for the validation. The HEMS data of these 16 households, which is of the sampling period $3 \mathrm{~min}$, is down-sampled to $15 \mathrm{~min}$ as the AMI data behaves and used for model training for the 5 appliances as mentioned. The difference of total power between HEMS meters and AMI meters have been calibrated and corrected. The model training process is basically based on the repository 'NeuralNILM' composed by Kelly et al at GitHub (https://github.com/JackKelly/neuralnilm).

\subsection{Questionnaire}

After the naïve model training of NILM estimation and classification, we have made a simple questionnaire on Google Form about the usage periods. 95\% of the AMI users have answered this questionnaire sheet. We did not investigate about the refrigerators since it should be always turned on in the public awareness. 
There are 2 issues to be concerned relating to behavioral science. First, it is impossible to know the daily behavior via questionnaire. These answers are to consider the "conjecture" from people toward themselves. People have their different standards on how often they use the appliances when they mark the period as "often used." Second, the answer may be wrong if people are ignorant about how they use their appliances. Therefore, this questionnaire should not be used naively as validation of the model training.

To make a sanity checking, we have compared the questionnaire with the data of HEMS to obtain the precision, as Table 2. We have designed a threshold of days for the classification: an appliance will be marked as "often used" in some period if it is turned on within that period in more days than the threshold days. For example, if someone watches television in 16 nights, then he is marked as "often uses television in the night." We adjust the threshold so that the sum of the precision of the prediction and the questionnaire obtains the maximum.

Table 1: An example of the questionnaire

\begin{tabular}{|l|l|}
\hline Columns & Answer \\
\hline User & cpchang@iii.org.tw \\
\hline Appliances & $\begin{array}{l}\text { air conditioner, refrigerator, washing machine, } \\
\text { bottle warmer }\end{array}$ \\
\hline usage of air conditioners & morning night \\
\hline usage of refrigerators & night \\
\hline usage of washing machines & afternoon, night \\
\hline usage of bottle warmers & morning afternoon, night \\
\hline usage of televisions & none \\
\hline
\end{tabular}

Table 2: Comparison between the questionnaire and the prediction toward NILM classification

\begin{tabular}{|l|l|l|l|}
\hline Appliances & $\begin{array}{l}\text { Precision of model } \\
\text { prediction }\end{array}$ & $\begin{array}{l}\text { Precision of } \\
\text { questionnaire }\end{array}$ & $\begin{array}{l}\text { Threshold days } \\
\text { of "Often used" }\end{array}$ \\
\hline television & 0.73 & 0.57 & 15 \\
\hline air conditioner & 0.62 & 0.73 & 10 \\
\hline bottle warmer & 0.61 & 0.79 & 15 \\
\hline washing machine & 0.89 & 0.67 & 6 \\
\hline
\end{tabular}

And we may observe that the questionnaire behaves better than the model prediction for the air conditioners and the bottle warmers. So, our next step is to tune the models for these 2 appliances.

\subsection{Tune models via Questionnaire}

As Figure 4, we choose to tune our model for AMI classification of the air conditioners and the bottle warmers. First, we train the model of classification from the HEMS and the AMI features. To make up the labels corresponding to AMI features, we impose the data of bottle warmers and the air conditioners from the questionnaire so that these features and labels can be used to train model as well. The obtained classifier model is used to tune the estimation percentage by encouraging or suppressing the ratio weight if it is determined to be turned on or off in this period, respectively. Specifically, if the television is to be determined on in some period with the NILM classification, then we raise the percentage estimation of television by $25 \%$ in that period. On the other hand, we lower the percentage estimation of television by $25 \%$ if it is determined to be off in that period. 


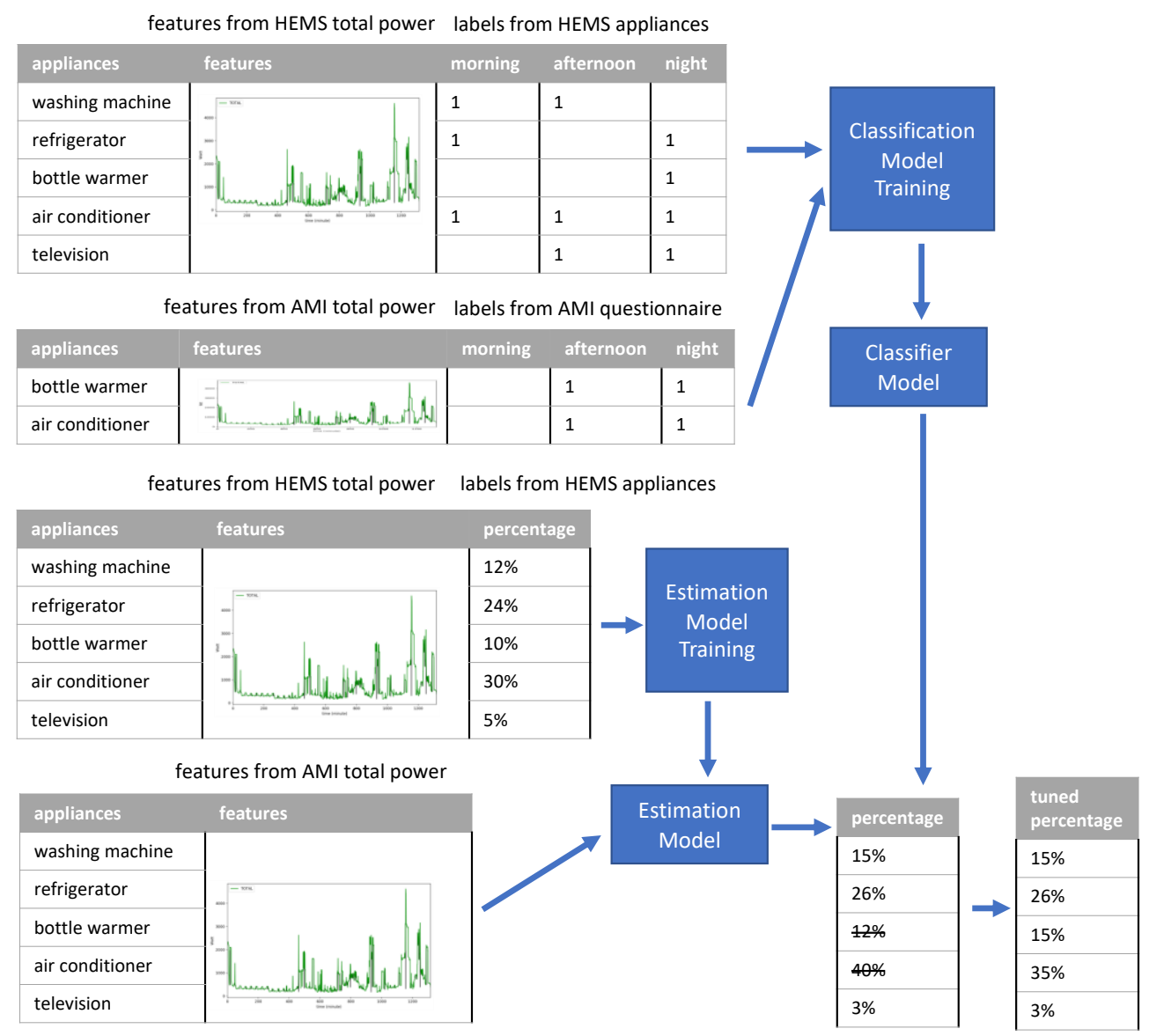

Figure 4: Questionnaire-tuned models for both classification and estimation.

Table 3 shows the prediction result tuned via questionnaire. We impose REITE (relative error in total energy) and the precision to be the measure for estimation and classification, respectively,

$$
\begin{aligned}
\text { REITE } & =\frac{\mid \text { predicted power consumption-actual power consumption } \mid}{\max (\text { predicted power consumption, actual power consumption })} \\
\text { Precision } & =\frac{\text { Number of periods } \text { in which this appliance is actually turned on }}{\text { Number of periods in which this appliance is predicted to be turned on }} .
\end{aligned}
$$

This improved result implies this low-cost-questionnaire-tuned strategy works.

Table 3: Prediction result

\begin{tabular}{|l|l|l|}
\hline \multicolumn{1}{|c|}{ Appliances } & Classification Precision & $\begin{array}{l}\text { Estimation Relative Error in Total } \\
\text { Energy }\end{array}$ \\
\hline refrigerator & 0.89 & 0.06 \\
\hline air conditioner & 0.73 & 0.09 \\
\hline air conditioner & 0.62 & 0.73 \\
\hline bottle warmer & 0.61 & 0.79 \\
\hline $\begin{array}{l}\text { washing } \\
\text { machine }\end{array}$ & 0.89 & 0.67 \\
\hline
\end{tabular}




\section{Conclusions}

For our empirical study, as Figure 5, we have investigated the NILM estimation and the classification on 150 AMI households in the same community by their smart meters. And we deployed a small amount HEMS smart plugs for the supervised model training. To improve the models, we have made free questionnaire and investigated the reliability of it. We have extracted the reliable part of this questionnaire to modify our own models, and demonstrated this lowpriced way works. This strategy costs few compared with thoroughly deployed smart plugs, so we believe it is an efficient way for power suppliers and the government. In September, 2020, we promoted our research in the Presidential Hackathon 2020, Taiwan. And we have won the elite award.

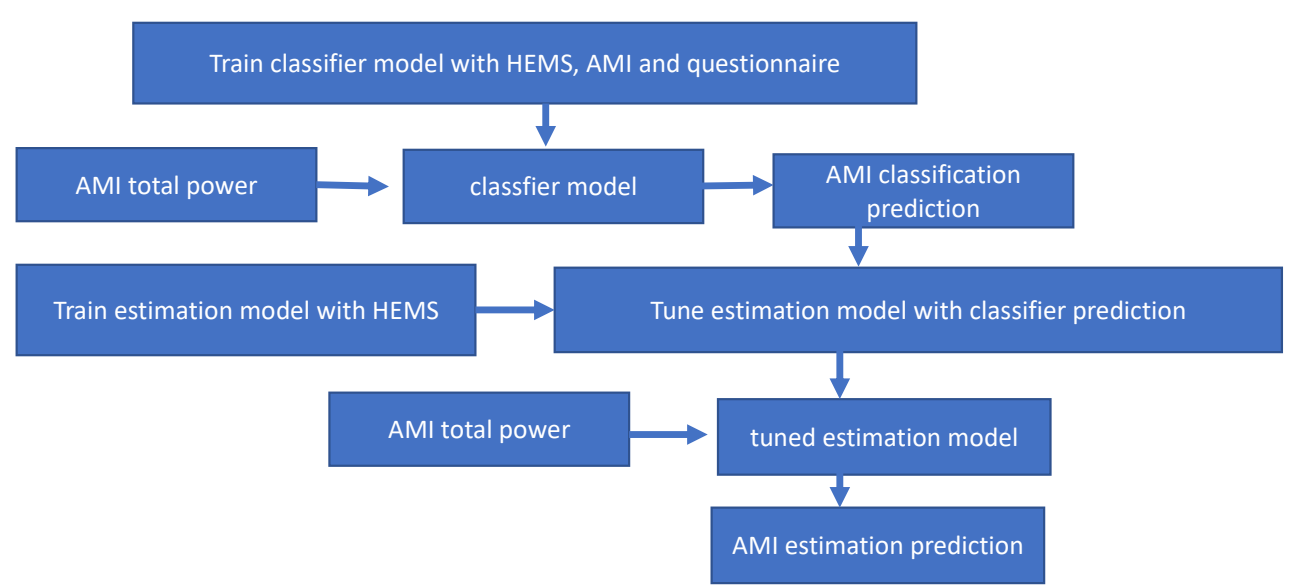

Figure 5: flow chart of questionnaire-tuned AMI classification and estimation

\section{ACKNOWLEDGEMENTS}

This work was supported by the Bureau of Energy, Ministry of Economic Affairs, Taiwan under the Grant No. 109-D0101-2.

\section{REFERENCES}

[1] G. W. Hart, "Nonintrusive Appliance Load Monitoring," Proc. IEEE, vol. 80, no. 12, pp. 1870-1891, 1992.

[2] J. Kelly and W. Knottenbelt, "Neural NILM: Deep neural networks applied to energy disaggregation,” BuildSys 2015 - Proc. 2nd ACM Int. Conf. Embed. Syst. Energy-Efficient Built, pp. 55-64, 2015.

[3] R. Bonfigli, A. Felicetti, E. Principi, M. Fagiani, S. Squartini, and F. Piazza, "Denoising autoencoders for Non-Intrusive Load Monitoring: Improvements and comparative evaluation," Energy Build., vol. 158, pp. 1461-1474, 2018.

[4] K. Basu, A. Hably, V. Debusschere, S. Bacha, J. Dirven, and A. Ovalle, "A comparative study of low sampling non-intrusive load disaggregation," Oct. 2016.

[5] F. Y. Chang and W. J. Ho, "An Analysis of Semi-Supervised Learning Approaches in Low-Rate Energy Disaggregation," in Proceedings - 2019 3rd International Conference on Smart Grid and Smart Cities, ICSGSC 2019, 2019, pp. 145-150. 


\section{Authors}

Chun-peng Chang obtained the Ph.D. degree of physics from National Tsing Hua University, Taiwan in 2014. Now he is a data scientist in the Institute for the Information Industry, Taiwan. His research is the analysis and prediction on energy consumption and generation via machine learning and deep learning.

(C) 2020 By AIRCC Publishing Corporation. This article is published under the Creative Commons Attribution (CC BY) license. 